\title{
Bipolar Intuitionistic M Fuzzy Group and Anti M Fuzzy Group.
}

\author{
K. Gunasekaran ${ }^{1}$, S. Nandakumar ${ }^{2}$, D. Gunaseelan ${ }^{3}$ \\ ${ }^{1,3}$ Ramanujan Research Centre, PG and Research Department of Mathematics, Government Arts \\ College (Autonomous), Kumbakonam-612002, Tamilnadu, India. \\ ${ }^{2}$ PG and Research Department of Mathematics, Government Arts College Ariyalur, Tamilnadu, India.
}

\begin{abstract}
The concept of a Bipolar intuitionistic M fuzzy group is a new algebraic structure of a bipolar intuitionistic M fuzzy subgroup of a M fuzzy group and anti M fuzzy group are defined and some related properties are investigated. The purpose of the study is to implement the fuzzy set theory and group theory of bipolar intuitionistic $M$ fuzzy subgroup of a $M$ fuzzy group and anti $M$ fuzzy group. The relation between of a bipolar intuitionistic $M$ fuzzy group and bipolar intuitionistic anti $M$ fuzzy group are established.
\end{abstract}

Keywords: $M$ fuzzy group, anti M fuzzy group, bipolar intuitionistic fuzzy set, bipolar intuitionistic M fuzzy group, bipolar intuitionistic anti M fuzzy group.

\section{Introduction}

The concept of fuzzy sets was initiated by L.A. Zadeh [13] then it has become a vigorous area of research in engineering, medical science, graph theory. Rosenfeld [12] gave the idea of fuzzy subgroups. Bipolar valued fuzzy sets was introduced by K.M. Lee [5] are an extension of fuzzy sets whose membership degree range is enlarged from the interval $[0,1]$ to $[-1,1]$. In a bipolar valued fuzzy set, the membership degree 0 means that the elements are irrelevant to the corresponding property, the membership degree $(0,1]$ indicates that elements somewhat satisfy the property and the membership degree[-1,0) indicates that elements somewhat satisfy the implicit counter property. The author W. R. Zhang [15] commenced the concept of bipolar fuzzy sets as a generalization of fuzzy sets in 1994. The author Mourad Oqla [6] commenced the concept of an intuitionistic anti M fuzzy group. Chakrabarthy and R.Nanda [1] investicated note on union and intersection of intuitionistic fuzzy sets. P.S. Das, A. Rajeshkumar [2,3] were analyzed fuzzy groups and level subgroups. R. Muthuraj [8,9] introduced the concept of bipolar fuzzy subgroup of a $M$ fuzzy group and bipolar anti $M$ fuzzy group. He was introduced the notion of an image and pre-image of a bipolar fuzzy subset of a bipolar fuzzy subgroup of a group and also discuss some of its properties of bipolar M fuzzy subgroup under $\mathrm{M}$ homomorphism and $\mathrm{M}$ anti homomorphism. We discuss some of its properties with bipolar intuitionistic $\mathrm{M}$ fuzzy subgroup of $M$ fuzzy group and anti $M$ fuzzy group are established under $M$ homomorphism and $M$ anti homomorphism.

\section{Preliminaries}

In this paper $G=(G, *)$ is a finite groups, e is the identity element of $\mathrm{G}$, and xy mean $\mathrm{x} * \mathrm{y}$ the fundamental definitions that will be used in the sequel.

Definition.2.1 Let $G$ be a non empty set, A bipolar intuitionistic fuzzy set (IFS) $A$ in $G$ is an object of the form $A=\left\{x, \mu_{A}^{+}(x), \mu_{A}^{-}(x), v_{A}^{+}(x), v_{A}^{-}(x) / x \in G\right\}$ where $\mu_{A}^{+}: G \rightarrow[0,1]$ and $v_{A}^{+}: G \rightarrow[0,1]$, $\mu_{A}^{-}: G \rightarrow[-1,0]$ and $v_{A}^{-}: G \rightarrow[-1,0]$ is called degree of positive membership, degree of negative membership and the degree of positive non membership, degree of negative non membership respectively.

Definition.2.2 [8] Let G be a group. A bipolar valued intuitionistic fuzzy set (IFS) A of G is called a bipolar intuitionistic fuzzy subgroup of $\mathrm{G}$, if for all $\mathrm{x}, \mathrm{y} \in \mathrm{G}$

$$
\begin{aligned}
& \text { i) } \mu_{A}^{+}(x y) \geq \min \left(\mu_{A}^{+}(x), \mu_{A}^{+}(y)\right) \text { and } v_{A}^{+}(x y) \leq \max \left(v_{A}^{+}(x), v_{A}^{+}(y)\right) \\
& \text { ii) } \mu_{A}^{-}(x y) \leq \max \left(\mu_{A}^{-}(x), \mu_{A}^{-}(y)\right) \text { and } v_{A}^{-}(x y) \geq \min \left(v_{A}^{-}(x), v_{A}^{-}(y)\right) \\
& \text { iii) } \mu_{A}^{+}\left(x^{-1}\right)=\mu_{A}^{+}(x), \mu_{A}^{-}\left(x^{-1}\right)=\mu_{A}^{-}(x) \text { and } v_{A}^{+}\left(x^{-1}\right)=v_{A}^{+}(x), v_{A}^{-}\left(x^{-1}\right)=v_{A}^{-}(x) .
\end{aligned}
$$




\section{Example.2.3}

$$
\mu_{A}^{+}(x)=\left\{\begin{array}{c}
0.7 \text { if } x=1 \\
0.6 \text { if } x=-1 \\
0.4 \text { if } x=i,-i
\end{array} ; v_{A}^{+}(x)=\left\{\begin{array}{c}
0.2 \text { if } x=1 \\
0.3 \text { if } x=-1 \\
0.5 \text { if } x=i,-i
\end{array} \text { and } \mu_{A}^{-}(x)=\left\{\begin{array}{l}
-0.8 \text { if } x=1 \\
-0.5 \text { if } x=-1 \\
-0.3 \text { if } x=i,-i
\end{array} ; v_{A}^{-}(x)=\left\{\begin{array}{l}
-0.1 \text { if } x=1 \\
-0.4 \text { if } x=-1 \\
-0.6 \text { if } x=i,-i
\end{array}\right.\right.\right.\right.
$$

Definition.2.4 [7] Let $G$ be a group. A bipolar valued IFS (or) bipolar IFS A of $G$ is called a bipolar intuitionistic anti fuzzy subgroup of $G$, if for all $x, y \in G$

$$
\begin{aligned}
& \text { i) } \mu_{A}^{+}(x y) \leq \max \left(\mu_{A}^{+}(x), \mu_{A}^{+}(y)\right) \text { and } v_{A}^{+}(x y) \geq \min \left(v_{A}^{+}(x), v_{A}^{+}(y)\right) \\
& \text { ii) } \mu_{A}^{-}(x y) \geq \min \left(\mu_{A}^{-}(x), \mu_{A}^{-}(y)\right) \text { and } v_{A}^{-}(x y) \leq \max \left(v_{A}^{-}(x), v_{A}^{-}(y)\right) \\
& \text { iii) } \mu_{A}^{+}\left(x^{-1}\right)=\mu_{A}^{+}(x), \mu_{A}^{-}\left(x^{-1}\right)=\mu_{A}^{-}(x) \text { and } v_{A}^{+}\left(x^{-1}\right)=v_{A}^{+}(x), v_{A}^{-}\left(x^{-1}\right)=v_{A}^{-}(x) .
\end{aligned}
$$

\section{Example.2.5}

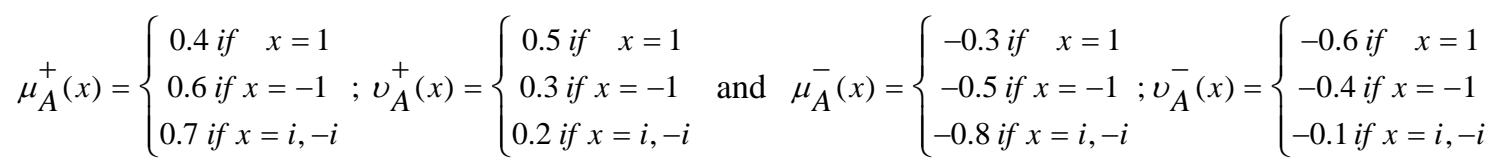

Definition.2.6 Let $G$ be an $M$ group and $A$ be a bipolar intuitionistic fuzzy subgroup of $G$, then $A$ is called a bipolar intuitionistic $M$ fuzzy group of $G$, if for all $x \in G$ and $m \in M$ then,

$$
\text { i) } \mu_{A}^{+}(m x) \geq \mu_{A}^{+}(x) \text { and } v_{A}^{+}(m x) \leq v_{A}^{+}(x) \text {. ii) } \mu_{A}^{-}(m x) \leq \mu_{A}^{-}(x) \text { and } v_{A}^{-}(m x) \geq v_{A}^{-}(x) .
$$

\section{Example.2.7}

Consider $1 \in M$

$$
\mu_{A}^{+}(x)=\left\{\begin{array}{c}
0.7 \text { if } x=1 \\
0.6 \text { if } x=-1 \\
0.4 \text { if } x=i,-i
\end{array} ; v_{A}^{+}(x)=\left\{\begin{array}{c}
0.2 \text { if } x=1 \\
0.3 \text { if } x=-1 \\
0.5 \text { if } x=i,-i
\end{array} \text { and } \mu_{A}^{-}(x)=\left\{\begin{array}{l}
-0.8 \text { if } x=1 \\
-0.5 \text { if } x=-1 \\
-0.3 \text { if } x=i,-i
\end{array} ; v_{A}^{-}(x)=\left\{\begin{array}{l}
-0.1 \text { if } x=1 \\
-0.4 \text { if } x=-1 \\
-0.6 \text { if } x=i,-i
\end{array}\right.\right.\right.\right.
$$

Definition.2.8 Let $\mathrm{G}$ be an $\mathrm{M}$ group and $\mathrm{A}$ be a bipolar intuitionistic anti fuzzy subgroup of $\mathrm{G}$, then $\mathrm{A}$ is called a bipolar intuitionistic anti $\mathrm{M}$ fuzzy group of $\mathrm{G}$, if for all $x \in G$ and $\mathrm{m} \in \mathrm{M}$ then,

$$
\text { i) } \mu_{A}^{+}(m x) \leq \mu_{A}^{+}(x) \text { and } v_{A}^{+}(m x) \geq v_{A}^{+}(x) \text {. ii) } \mu_{A}^{-}(m x) \geq \mu_{A}^{-}(x) \text { and } v_{A}^{-}(m x) \leq v_{A}^{-}(x) \text {. }
$$

\section{Example.2.9}

\section{Consider $1 \in M$}

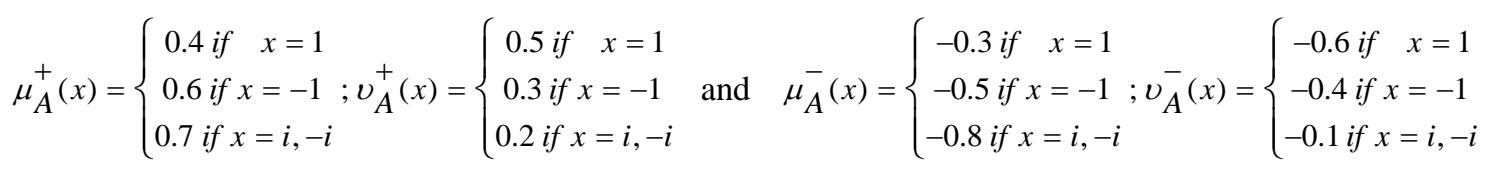

Theorem.2.10 If $\mathrm{A}$ and $\mathrm{B}$ are bipolar intuitionistic $\mathrm{M}$ fuzzy group of $\mathrm{G}$, then $A \cap B$ is a bipolar intuitionistic $M$ fuzzy group of $\mathrm{G}$.

Proof Consider $m \in M$ and $x \in A \cap B$ implies $x \in A, x \in B$ 
Consider $\mu_{A \cap B}^{+}(m x)=\min \left(\mu_{A}^{+}(m x), \mu_{B}^{+}(m x)\right) \geq \min \left(\mu_{A}^{+}(x), \mu_{B}^{+}(x)\right)=\mu_{A \bigcap B}^{+}(x)$.

Therefore $\mu_{A \bigcap B}^{+}(m x) \geq \mu_{A \bigcap B}^{+}(x)$.

Consider $v_{A \cap B}^{+}(m x)=\max \left(v_{A}^{+}(m x), v_{B}^{+}(m x)\right) \leq \max \left(v_{A}^{+}(x), v_{B}^{+}(x)\right)=v_{A \cap B}^{+}(x)$.

Therefore $v_{A \cap B}^{+}(m x) \leq v_{A \cap B}^{+}(x)$.

Consider $\mu_{A \bigcap B}^{-}(m x)=\max \left(\mu_{A}^{-}(m x), \mu_{B}^{-}(m x)\right) \leq \max \left(\mu_{A}^{-}(x), \mu_{B}^{-}(x)\right)=\mu_{A \bigcap B}^{-}(x)$.

Therefore $\mu_{A \bigcap B}^{-}(m x) \leq \mu_{A \bigcap B}^{-}(x)$.

Consider $v_{A \cap B}^{-}(m x)=\min \left(v_{A}^{-}(m x), v_{B}^{-}(m x)\right) \geq \min \left(v_{A}^{-}(x), v_{B}^{-}(x)\right)=v_{A \cap B}^{-}(x)$.

Therefore $v_{A \cap B}^{-}(m x) \geq v_{A \cap B}^{-}(x)$.

Therefore $A \cap B$ is a bipolar intuitionistic M fuzzy group of $\mathrm{G}$

Theorem.2.11 If $\mathrm{A}$ is a bipolar intuitionistic $\mathrm{M}$ fuzzy group of $\mathrm{G}$, then $\overline{\bar{A}}=A$ is also a bipolar intuitionistic M fuzzy group of $\mathrm{G}$.

Proof $\quad$ Let $m \in M$ and $x \in A$

$$
\begin{gathered}
\text { Consider } \mu_{\overline{\bar{A}}}^{+}(m x)=v_{\bar{A}}^{+}(m x)=\mu_{A}^{+}(m x) \geq \mu_{A}^{+}(x) \text {. Therefore } \mu_{\overline{\bar{A}}}^{+}(m x) \geq \mu_{A}^{+}(x) . \\
\text { Consider } v_{\overline{\bar{A}}}^{+}(m x)=\mu_{\bar{A}}^{+}(m x)=v_{A}^{+}(m x) \leq v_{A}^{+}(x) . \quad \text { Therefore } v_{\overline{\bar{A}}}^{+}(m x) \leq v_{A}^{+}(x) . \\
\text { Consider } \mu_{\overline{\bar{A}}}^{-}(m x)=v_{\bar{A}}^{-}(m x)=\mu_{A}^{-}(m x) \leq \mu_{A}^{-}(x) . \text { Therefore } \mu_{\overline{\bar{A}}}^{-}(m x) \leq \mu_{A}^{-}(x) . \\
\text { Consider } v_{\overline{\bar{A}}}^{-}(m x)=\mu_{\bar{A}}^{-}(m x)=v_{A}^{-}(m x) \geq v_{A}^{-}(x) . \quad \text { Therefore } v_{\overline{\bar{A}}}^{-}(m x) \geq v_{A}^{-}(x) . \\
\text { Therefore } \overline{\bar{A}}=A \text { is a bipolar intuitionistic M fuzzy group of G. }
\end{gathered}
$$

Theorem.2.12 Union of any two bipolar intuitionistic $M$ fuzzy group is also a bipolar intuitionistic M fuzzy group if either is contained in the other.

Proof Let $\mathrm{A}$ and $\mathrm{B}$ be a bipolar intuitionistic M fuzzy group of $\mathrm{G}$.

To prove that $A \cup B$ is a bipolar intuitionistic M fuzzy group of $\mathrm{G}$ if $A \subseteq B($ or $) B \subseteq A$

If $\quad A \subseteq B \Rightarrow A \cup B=B$ (or) $B \subseteq A \Rightarrow A \cup B=A$

Let $m \in M \& x \in A \cup B$

Consider $\mu_{A \cup B}^{+}(m x)=\max \left(\mu_{A}^{+}(m x), \mu_{B}^{+}(m x)\right) \geq \max \left(\mu_{A}^{+}(x), \mu_{B}^{+}(x)\right)=\mu_{A \cup B}^{+}(x)$.

Therefore $\mu_{A \cup B}^{+}(m x) \geq \mu_{A \cup B}^{+}(x)$.

Consider $v_{A \cup B}^{+}(m x)=\min \left(v_{A}^{+}(m x), v_{B}^{+}(m x)\right) \leq \min \left(v_{A}^{+}(x), v_{B}^{+}(x)\right)=v_{A \cup B}^{+}(x)$.

Therefore $v_{A \cup B}^{+}(m x) \leq v_{A \cup B}^{+}(x)$. 
Consider $\mu_{A \cup B}^{-}(m x)=\min \left(\mu_{A}^{-}(m x), \mu_{B}^{-}(m x)\right) \leq \min \left(\mu_{A}^{-}(x), \mu_{B}^{-}(x)\right)=\mu_{A \cup B}^{-}(x)$.

Therefore $\mu_{A \cup B}^{-}(m x) \leq \mu_{A \cup B}^{-}(x)$.

Consider $v_{A \cup B}^{-}(m x)=\max \left(v_{A}^{-}(m x), v_{B}^{-}(m x)\right) \geq \max \left(v_{A}^{-}(x), v_{B}^{-}(x)\right)=v_{A \cup B}^{-}(x)$.

Therefore $v_{A \cup B}^{-}(m x) \geq v_{A \cup B}^{-}(x)$.

Hence union of any two bipolar intuitionistic $M$ fuzzy group is also a bipolar intuitionistic $M$ fuzzy group if either is contained in the other.

Theorem.2.13 If $\mathrm{A}$ is a bipolar intuitionistic anti $\mathrm{M}$ fuzzy group of $\mathrm{G}$, then $\overline{\bar{A}}=A$ is also a bipolar intuitionistic anti M fuzzy group of $\mathrm{G}$.

Proof Consider $m \in M$ and $x \in A$

Consider $\mu_{\overline{\bar{A}}}^{+}(m x)=v_{\bar{A}}^{+}(m x)=\mu_{A}^{+}(m x) \leq \mu_{A}^{+}(x)$. Therefore $\mu_{\bar{A}}^{+}(m x) \leq \mu_{A}^{+}(x)$.

Consider $v_{\overline{\bar{A}}}^{+}(m x)=\mu \frac{+}{\bar{A}}(m x)=v_{A}^{+}(m x) \geq v_{A}^{+}(x)$. Therefore $v_{\overline{\bar{A}}}^{+}(m x) \geq v_{A}^{+}(x)$.

Consider $\mu_{\overline{\bar{A}}}^{-}(m x)=v_{\bar{A}}^{-}(m x)=\mu_{A}^{-}(m x) \geq \mu_{A}^{-}(x)$. Therefore $\mu_{\overline{\bar{A}}}^{-}(m x) \geq \mu_{A}^{-}(x)$.

Consider $v_{\overline{\bar{A}}}^{-}(m x)=\mu \overline{\bar{A}}_{\bar{A}}^{-}(m x)=v_{\bar{A}}^{-}(m x) \leq v_{A}^{-}(x)$. Therefore $v_{\overline{\bar{A}}}^{-}(m x) \leq v_{\bar{A}}^{-}(x)$.

Therefore $\overline{\bar{A}}=A$ is a bipolar intuitionistic anti M fuzzy group of G.

Theorem.2.14 Union of any two bipolar intuitionistic anti $M$ fuzzy group is also a bipolar intuitionistic anti $\mathrm{M}$ fuzzy group if either is contained in the other.

Proof Let $\mathrm{A}$ and $\mathrm{B}$ be a bipolar intuitionistic anti $\mathrm{M}$ fuzzy group of $\mathrm{G}$. To prove that $A \cup B$ is also a bipolar intuitionistic anti $\mathrm{M}$ fuzzy group of $\mathrm{G}$ if $A \subseteq B(o r) B \subseteq A$

If $A \subseteq B \Rightarrow A \bigcup B=B$ (or) $B \subseteq A \Rightarrow A \bigcup B=A$.

Consider $m \in M$ and $x \in A \cup B$.

Consider $\mu_{A \cup B}^{+}(m x)=\max \left(\mu_{A}^{+}(m x), \mu_{B}^{+}(m x)\right) \leq \max \left(\mu_{A}^{+}(x), \mu_{B}^{+}(x)\right)=\mu_{A \cup B}^{+}(x)$.

Therefore $\mu_{A \cup B}^{+}(m x) \leq \mu_{A \cup B}^{+}(x)$.

Consider $v_{A \cup B}^{+}(m x)=\min \left(v_{A}^{+}(m x), v_{B}^{+}(m x)\right) \geq \min \left(v_{A}^{+}(x), v_{B}^{+}(x)\right)=v_{A \cup B}^{+}(x)$.

Therefore $v_{A \cup B}^{+}(m x) \geq v_{A \cup B}^{+}(x)$.

Consider $\mu_{A \cup B}^{-}(m x)=\min \left(\mu_{A}^{-}(m x), \mu_{B}^{-}(m x)\right) \geq \min \left(\mu_{A}^{-}(x), \mu_{B}^{-}(x)\right)=\mu_{A \cup B}^{-}(x)$.

Therefore $\mu_{A \cup B}^{-}(m x) \geq \mu_{A \cup B}^{-}(x)$.

Consider $v_{A \cup B}^{-}(m x)=\max \left(v_{A}^{-}(m x), v_{B}^{-}(m x)\right) \leq \max \left(v_{A}^{-}(x), v_{B}^{-}(x)\right)=v_{A \cup B}^{-}(x)$.

Therefore $v_{A \cup B}^{-}(m x) \leq v_{A \cup B}^{-}(x)$.

Therefore union of any two bipolar intuitionistic anti $M$ fuzzy group is also a bipolar intuitionistic anti M fuzzy group if either is contained in the other. 


\section{Some Result Based On Bipolar Intuitionistic M Fuzzy Group And Anti M Fuzzy Group Of G.}

Theorem.3.1 Let $\mu$ and $v$ be a bipolar intuitionistic fuzzy subset of an M fuzzy group then $\mu=\left(\mu^{+}, \mu^{-}\right)$is a bipolar intuitionistic $\mathrm{M}$ fuzzy group of $\mathrm{G}$ if and only if $v=\left(v^{+}, v^{-}\right)$is a bipolar intuitionistic anti $M$ fuzzy group of $\mathrm{G}$.

Proof Let $\mu=\left(\mu^{+}, \mu^{-}\right)$be a bipolar intuitionistic $\mathrm{M}$ fuzzy group of $\mathrm{G}$. To prove $v=\left(v^{+}, v^{-}\right)$is a bipolar intuitionistic anti $\mathrm{M}$ fuzzy group of $\mathrm{G}$.

$$
\text { i) } \begin{aligned}
\mu^{+}(x y) \geq \min \left\{\mu^{+}(x), \mu^{+}(y)\right\} & \Leftrightarrow 1-v^{+}(x y) \geq \min \left\{1-v^{+}(x), 1-v^{+}(y)\right\} \\
& \Leftrightarrow v^{+}(x y) \leq 1-\min \left\{1-v^{+}(x), 1-v^{+}(y)\right\} \\
& \Leftrightarrow v^{+}(x y) \leq \max \left\{v^{+}(x), v^{+}(y)\right\} .
\end{aligned}
$$

Therefore $\mu^{+}(x y) \geq \min \left\{\mu^{+}(x), \mu^{+}(y)\right\} \Leftrightarrow v^{+}(x y) \leq \max \left\{v^{+}(x), v^{+}(y)\right\}$.

ii) $\mu^{-}(x y) \leq \max \left\{\mu^{-}(x), \mu^{-}(y)\right\} \Leftrightarrow-1-v^{-}(x y) \leq \max \left\{-1-v^{-}(x),-1-v^{-}(y)\right\}$

$$
\begin{aligned}
& \Leftrightarrow v^{-}(x y) \geq-1-\max \left\{-1-v^{-}(x),-1-v^{-}(y)\right\} \\
& \Leftrightarrow v^{-}(x y) \geq \min \left\{v^{-}(x), v^{-}(y)\right\} .
\end{aligned}
$$

Therefore $\mu^{-}(x y) \leq \max \left\{\mu^{-}(x), \mu^{-}(y)\right\} \Leftrightarrow v^{-}(x y) \geq \min \left\{v^{-}(x), v^{-}(y)\right\}$.

iii) $\mu^{+}\left(x^{-1}\right)=\mu^{+}(x) \Leftrightarrow 1-v^{+}\left(x^{-1}\right)=1-v^{+}(x) \Leftrightarrow v^{+}\left(x^{-1}\right)=v^{+}(x)$. and

$\mu^{-}\left(x^{-1}\right)=\mu^{-}(x) \Leftrightarrow-1-v^{-}\left(x^{-1}\right)=-1-v^{-}(x) \Leftrightarrow v^{-}\left(x^{-1}\right)=v^{-}(x)$.

iv) $\mu^{+}(m x) \geq \mu^{+}(x) \Leftrightarrow 1-\mu^{+}(m x) \leq 1-\mu^{+}(x) \Leftrightarrow v^{+}(m x) \leq v^{+}(x)$.

Therefore $\mu^{+}(m x) \geq \mu^{+}(x)$ and $v^{+}(m x) \leq v^{+}(x)$.

v) $\mu^{-}(m x) \leq \mu^{-}(x) \Leftrightarrow-1-\mu^{-}(m x) \geq-1-\mu^{-}(x) \Leftrightarrow v^{-}(m x) \geq v^{-}(x)$.

Therefore $\mu^{-}(m x) \leq \mu^{-}(x)$ and $v^{-}(m x) \geq v^{-}(x)$.

Therefore $\mu=\left(\mu^{+}, \mu^{-}\right)$is a bipolar intuitionistic M fuzzy group of $\mathrm{G}$ if and only if $v=\left(v^{+}, v^{-}\right)$is a bipolar intuitionistic anti M fuzzy group of $\mathrm{G}$.

Definition.3.2 [9] Let $\mathrm{f}$ and $\mathrm{g}$ be a mapping from a group $G_{1}$ to a group $G_{2}$. Let $\mu=\left(\mu^{+}, \mu^{-}\right)$ and $\phi=\left(\phi^{+}, \phi^{-}\right)$and $v=\left(v^{+}, v^{-}\right), \psi=\left(\psi^{+}, \psi^{-}\right)$are bipolar intuitionistic fuzzy subset in $G_{1}$ and $G_{2}$ respectively, then the image $f(\mu)$ and $g(v)$ is a bipolar intuitionistic fuzzy subset is defined by $f(\mu)=\left(f(\mu)^{+}, f(\mu)^{-}\right)$and $g(v)=\left(g(v)^{+}, g(v)^{-}\right)$of $G_{2}$ for all $\mathrm{u}, \mathrm{v} \in G_{2}$. 


$$
f\left(\mu^{+}\right)(u)=\max \left\{\mu^{+}(x) ; x \in f^{-1}(u)\right\} \text { if } f^{-1}(u) \neq \varphi, 0 \text { and } g\left(v^{+}\right)(v)=\min \left\{v^{+}(x) ; x \in g^{-1}(v)\right\} \text { if }
$$
$g^{-1}(v) \neq \varphi, 0$ and $f\left(\mu^{-}\right)(u)=\min \left\{\mu^{-}(x) ; x \in f^{-1}(u)\right\}$, if $f^{-1}(u) \neq \varphi, 0 \quad g\left(v^{-}\right)(v)=\max \left\{v^{-}(x)\right.$; $\left.x \in g^{-1}(v)\right\}$ if $g^{-1}(v) \neq \varphi, 0$. The preimage $f^{-1}(\phi)$ is under $f$ and $g^{-1}(\psi)$ is under $\mathrm{g}$ is defined by the bipolar intuitionistic fuzzy subset of $G_{1}$ for all $x \in G_{1}, \quad\left(f^{-1}(\phi)^{+}\right)(x)=\phi^{+}(f(x))$; $\left(f^{-1}(\phi)^{-}\right)(x)=\phi^{-}(f(x))$ and $\left(g^{-1}(\psi)^{+}\right)(x)=\psi^{+}(g(x)) ; \quad\left(g^{-1}(\psi)^{-}\right)(x)=\psi^{-}(g(x))$.

Definition.3.3 [8] Let $G_{1}$ and $G_{2}$ be any two bipolar intuitionistic $\mathrm{M}$ groups then the function f: $G_{1} \rightarrow G_{2}$ and g: $G_{1} \rightarrow G_{2}$ is said to be an intuitionistic M homomorphism if,

i) $\mathrm{f}(\mathrm{xy})=\mathrm{f}(\mathrm{x}) \mathrm{f}(\mathrm{y})$ for all $x, y \in G_{1}$

ii) $\mathrm{f}(\mathrm{mx})=\mathrm{mf}(\mathrm{x})$ for all $m \in M$ and $x \in G_{1}$

iii) $\mathrm{g}(\mathrm{xy})=\mathrm{g}(\mathrm{x}) \mathrm{g}(\mathrm{y})$ for all $x, y \in G_{1}$

iv) $\mathrm{g}(\mathrm{mx})=\mathrm{mg}(\mathrm{x})$ for all $m \in M$ and $x \in G_{1}$.

Definition.3.4 [8] Let $G_{1}$ and $G_{2}$ be any two bipolar intuitionistic $M$ groups (not necessarily commutative) then the function $f: G_{1} \rightarrow G_{2}$ and $\mathrm{g}: G_{1} \rightarrow G_{2}$ is said to be an intuitionistic $\mathrm{M}$ anti homomorphism if,
i) $\mathrm{f}(\mathrm{xy})=\mathrm{f}(\mathrm{x}) \mathrm{f}(\mathrm{y})$ for all $x, y \in G_{1}$
ii) $\mathrm{f}(\mathrm{mx})=\mathrm{m} \mathrm{f}(\mathrm{x})$ for all $m \in M$ and $x \in G_{1}$
iii) $\mathrm{g}(\mathrm{xy})=\mathrm{g}(\mathrm{x}) \mathrm{g}(\mathrm{y})$ for all $x, y \in G_{1}$
iv) $\mathrm{g}(\mathrm{mx})=\mathrm{mg}(\mathrm{x})$ for all $m \in M$ and $x \in G_{1}$.

Theorem.3.5 Let $\mathrm{f}$ and $\mathrm{g}$ be an intuitionistic $\mathrm{M}$ homomorphism from an $\mathrm{M}$ fuzzy group of $G_{1}$ onto an $\mathrm{M}$ fuzzy group of $G_{2}$. If $\mu=\left(\mu^{+}, \mu^{-}\right)$is a bipolar intuitionistic $\mathrm{M}$ fuzzy group of $G_{1}$ then $f(\mu)$ the image of $\mu$ under $\mathrm{f}$ is a bipolar intuitionistic $\mathrm{M}$ fuzzy group of $G_{2}$ if and only if $v=\left(v^{+}, v^{-}\right)$is a bipolar intuitionistic anti $\mathrm{M}$ fuzzy group of $G_{1}$ then $g(v)$ is the image of $v$ under $g$ is a bipolar intuitionistic anti $\mathrm{M}$ fuzzy group of $G_{2}$.

Proof Let $f: G_{1} \rightarrow G_{2}$ and $g: G_{1} \rightarrow G_{2}$ be an intuitionistic M homomorphism.

Let $\mu=\left(\mu^{+}, \mu^{-}\right)$and $v=\left(v^{+}, v^{-}\right)$is a bipolar intuitionistic $\mathrm{M}$ fuzzy group and bipolar intuitionistic anti $\mathrm{M}$ fuzzy group of $G_{1}$. To prove a bipolar intuitionistic fuzzy subset $f(\mu)=\left(f(\mu)^{+}, f(\mu)^{-}\right)$and $g(v)=\left(g(v)^{+}, g(v)^{-}\right)$on $G_{2}$ is a bipolar intuitionistic M fuzzy group and bipolar intuitionistic anti $\mathrm{M}$ fuzzy group.

Let $u, v \in G_{2}$ since $\mathrm{f}$ is a intuitionistic $\mathrm{M}$ homomorphism and so there exist $x, y \in G_{1}$ such that $\mathrm{f}(\mathrm{x})=\mathrm{u} \& \mathrm{f}(\mathrm{y})=\mathrm{v}$ it follows that $x y \in f^{-1}(u v)$. We have to prove that $\mathrm{g}$ is an intuitionistic $\mathrm{M}$ homomorphism so there exist $x, y \in G_{1}$ such that $\mathrm{g}(\mathrm{x})=\mathrm{u} \& \mathrm{~g}(\mathrm{y})=\mathrm{v}$ it follows that $x y \in g^{-1}(u v)$. 
i) $f(\mu)^{+}(u v)=\max \left\{\mu^{+}(z): z=x y \in f^{-1}(u v)\right\}$

$$
\begin{aligned}
& \geq \max \left\{\min \left\{\mu^{+}(x), \mu^{+}(y)\right\}: x \in f^{-1}(u), y \in f^{-1}(v)\right\} \\
& =\min \left\{f(\mu)^{+}(u), f(\mu)^{+}(v)\right\} .
\end{aligned}
$$

Therefore $f(\mu)^{+}(u v) \geq \min \left\{f(\mu)^{+}(u), f(\mu)^{+}(v)\right\}$

$$
\begin{aligned}
& \Leftrightarrow 1-g(v)^{+}(u v) \geq \min \left\{\left(1-g(v)^{+}\right)(u),\left(1-g(v)^{+}\right)(v)\right\} \\
& \Leftrightarrow g(v)^{+}(u v) \leq 1-\min \left\{\left(1-g(v)^{+}\right)(u),\left(1-g(v)^{+}\right)(v)\right\} \\
& \Leftrightarrow g(v)^{+}(u v) \leq \max \left\{g(v)^{+}(u), g(v)^{+}(v)\right\} .
\end{aligned}
$$

Hence $f(\mu)^{+}(u v) \geq \min \left\{f(\mu)^{+}(u), f(\mu)^{+}(v)\right\}$

$$
\Leftrightarrow g(v)^{+}(u v) \leq \max \left\{g(v)^{+}(u), g(v)^{+}(v)\right\} .
$$

ii) $f(\mu)^{-}(u v)=\max \left\{\mu^{-}(z): z=x y \in f^{-1}(u v)\right\}$

$$
\begin{aligned}
& \leq \max \left\{\max \left\{\mu^{-}(x), \mu^{-}(y)\right\}: x \in f^{-1}(u), y \in f^{-1}(v)\right\} \\
& =\max \left\{f(\mu)^{-}(u), f(\mu)^{-}(v)\right\} .
\end{aligned}
$$

Therefore $f(\mu)^{-}(u v) \leq \max \left\{f(\mu)^{-}(u), f(\mu)^{-}(v)\right\}$

$$
\begin{aligned}
& \Leftrightarrow\left(-1-g(v)^{-}\right)(u v) \leq \max \left\{\left(-1-g(v)^{-}\right)(u),\left(-1-g(v)^{-}\right)(v)\right\} \\
& \Leftrightarrow g(v)^{-}(u v) \geq-1-\max \left\{\left(-1-g(v)^{-}\right)(u),\left(-1-g(v)^{-}\right)(v)\right\} \\
& \Leftrightarrow g(v)^{-}(u v) \geq \min \left\{g(v)^{-}(u), g(v)^{-}(v)\right\} .
\end{aligned}
$$

Hence $f(\mu)^{-}(u v) \leq \max \left\{f(\mu)^{-}(u), f(\mu)^{-}(v)\right\}$

$$
\Leftrightarrow g(v)^{-}(u v) \geq \min \left\{g(v)^{-}(u), g(v)^{-}(v)\right\} .
$$

iii) Now $f(\mu)^{+}\left(u^{-1}\right)=\max \left\{\mu^{+}(x): x \in f^{-1}\left(u^{-1}\right)\right\}=\max \left\{\mu^{+}\left(x^{-1}\right): x^{-1} \in f^{-1}(u)\right\}$

$$
=f(\mu)^{+}(u)
$$

Therefore $f(\mu)^{+}\left(u^{-1}\right)=f(\mu)^{+}(u) \Leftrightarrow\left(1-g(v)^{+}\right)\left(u^{-1}\right)=\left(1-g(v)^{+}\right)(u)$

$$
\Leftrightarrow g(v)^{+}\left(u^{-1}\right)=g(v)^{+}(u) .
$$

Hence $f(\mu)^{+}\left(u^{-1}\right)=\left(f(\mu)^{+}(u) \Leftrightarrow g(v)^{+}\left(u^{-1}\right)=g(v)^{+}(u)\right.$.

iv) $f(\mu)^{-}\left(u^{-1}\right)=\min \left\{\mu^{-}(x): x \in f^{-1}\left(u^{-1}\right)\right\}=\min \left\{\mu^{-}\left(x^{-1}\right): x^{-1} \in f^{-1}(u)\right\}$

$$
=f(\mu)^{-}(u) \text {. }
$$

Therefore $f(\mu)^{-}\left(u^{-1}\right)=f(\mu)^{-}(u) \Leftrightarrow\left(-1-g(v)^{-}\right)\left(u^{-1}\right)=\left(-1-g(v)^{-}\right)(u)$

$$
\Leftrightarrow g(v)^{-}\left(u^{-1}\right)=g(v)^{-}(u) .
$$


Hence $\quad f(\mu)^{-}\left(u^{-1}\right)=f(\mu)^{-}(u) \Leftrightarrow g(v)^{-}\left(u^{-1}\right)=g(v)^{-}(u)$

Therefore $f(\mu)$ and $g(v)$ is a bipolar fuzzy subgroup of $G_{2}$.

v) Let $m \in M$ and $u \in G_{2}$,

$$
f(\mu)^{+}(m u) \geq \max \left\{\mu^{+}(x): x \in f^{-1}(u)\right\}=f\left(\mu^{+}\right)(u) .
$$

Therefore $f(\mu)^{+}(m u) \geq f(\mu)^{+}(u) \Leftrightarrow\left(1-g(v)^{+}\right)(m u) \geq\left(1-g(v)^{+}\right)(u)$

$$
\Leftrightarrow g(v)^{+}(m u) \leq g(v)^{+}(u) .
$$

Hence $f(\mu)^{+}(m u) \geq f(\mu)^{+}(u) \Leftrightarrow g(v)^{+}(m u) \leq g(v)^{+}(u)$.

vi) $f(\mu)^{-}(m u) \leq \min \left\{\mu^{-}(x): x \in f^{-1}(u)\right\}=f\left(\mu^{-}\right)(u)$.

Therefore $f(\mu)^{-}(m u) \leq f(\mu)^{-}(u) \Leftrightarrow\left(-1-g(v)^{-}\right)(m u) \leq\left(-1-g(v)^{-}\right)(u)$

$$
\Leftrightarrow g(v)^{-}(m u) \geq g(v)^{-}(u) \text {. }
$$

Hence $f(\mu)^{-}(m u) \leq f(\mu)^{-}(u) \Leftrightarrow g(v)^{-}(m u) \geq g(v)^{-}(u)$.

Therefore if $\mu$ be a bipolar intuitionistic M fuzzy group of $G_{1}$ then $f(\mu)$ is a bipolar intuitionistic $\mathrm{M}$ fuzzy group of $G_{2}$ if and only if $v$ be a bipolar intuitionistic anti $\mathrm{M}$ fuzzy group of $G_{1}$ then $g(v)$ be a bipolar intuitionistic anti $\mathrm{M}$ fuzzy group of $G_{2}$.

Theorem.3.6 The $\mathrm{M}$ homomorphic preimage of a bipolar intuitionistic $\mathrm{M}$ fuzzy group of $G_{2}$ is a bipolar intuitionistic $\mathrm{M}$ fuzzy group of $G_{1}$ if and only if $\mathrm{M}$ homomorphic preimage of a bipolar intuitionistic anti $\mathrm{M}$ fuzzy group of $G_{2}$ is a bipolar intuitionistic anti $\mathrm{M}$ fuzzy group of $G_{1}$.

Proof Let $f: G_{1} \rightarrow G_{2}$ and $g: G_{1} \rightarrow G_{2}$ be an intuitionistic M homomorphism. let $\phi=\left(\phi^{+}, \phi^{-}\right)$is a bipolar intuitionistic $\mathrm{M}$ fuzzy group of $G_{2}$ and $\psi=\left(\psi^{+}, \psi^{-}\right)$is a bipolar intuitionistic anti $\mathrm{M}$ fuzzy group of $G_{2}$,to prove a bipolar fuzzy subset $\mu=\left(\mu^{+}, \mu^{-}\right)$and $v=\left(v^{+}, v^{-}\right)$on $G_{1}$ is a bipolar intuitionistic $M$ fuzzy group and bipolar intuitionistic anti $M$ fuzzy group where $\mu=f^{-1}(\phi) \& v=g^{-1}(\psi)$

i) Consider $x, y \in G_{1}$

$$
\begin{aligned}
\left(f^{-1}(\phi)\right)^{+}(x y) & =\phi^{+}(f(x y)) \\
& \geq \min \left\{\phi^{+}(f(x)), \phi^{+}(f(y))\right\} \\
& =\min \left\{\left(f^{-1}(\phi)\right)^{+}(x),\left(f^{-1}(\phi)\right)^{+}(y)\right\} .
\end{aligned}
$$

Therefore $\left(f^{-1}(\phi)\right)^{+}(x y) \geq \min \left\{\left(f^{-1}(\phi)\right)^{+}(x),\left(f^{-1}(\phi)\right)^{+}(y)\right\}$. 


$$
\begin{aligned}
\Leftrightarrow\left(g^{-1}(\psi)\right)^{+}(x y) & =\psi^{+}(g(x y)) \leq \max \left\{\psi^{+}(g(x)), \psi^{+}(g(y))\right\} \\
& =\max \left\{\left(g^{-1}(\psi)\right)^{+}(x),\left(g^{-1}(\psi)\right)^{+}(y)\right\} .
\end{aligned}
$$

Hence $\quad\left(f^{-1}(\phi)\right)^{+}(x y) \geq \min \left\{\left(f^{-1}(\phi)\right)^{+}(x),\left(f^{-1}(\phi)\right)^{+}(y)\right.$

$$
\Leftrightarrow\left(g^{-1}(\psi)\right)^{+}(x y) \leq \max \left\{\left(g^{-1}(\psi)\right)^{+}(x),\left(g^{-1}(\psi)\right)^{+}(y)\right\} .
$$

ii) Let $x, y \in G_{1} \quad\left(f^{-1}(\phi)\right)^{-}(x y)=\phi^{-}(f(x y)) \leq \max \left\{\phi^{-}(f(x)), \phi^{-}(f(y))\right\}$

$$
=\max \left\{\left(f^{-1}(\phi)\right)^{-}(x),\left(f^{-1}(\phi)\right)^{-}(y)\right\} .
$$

Therefore $\left(f^{-1}(\phi)\right)^{-}(x y) \leq \max \left\{\left(f^{-1}(\phi)\right)^{-}(x),\left(f^{-1}(\phi)\right)^{-}(y)\right\}$

$$
\begin{aligned}
\Leftrightarrow\left(g^{-1}(\psi)\right)^{-}(x y) & =\psi^{-}(g(x y)) \\
& \geq \min \left\{\psi^{-}(g(x)), \psi^{-}(g(y))\right\} \\
& =\min \left\{\left(g^{-1}(\psi)\right)^{-}(x),\left(g^{-1}(\psi)\right)^{-}(y)\right\} .
\end{aligned}
$$

Hence $\quad\left(f^{-1}(\phi)\right)^{-}(x y) \leq \max \left\{\left(f^{-1}(\phi)\right)^{-}(x),\left(f^{-1}(\phi)\right)^{-}(y)\right.$

$$
\Leftrightarrow\left(g^{-1}(\psi)\right)^{-}(x y) \geq \min \left\{\left(g^{-1}(\psi)\right)^{-}(x),\left(g^{-1}(\psi)\right)^{-}(y)\right\}
$$

iii) Consider $x \in G_{1}$

$$
\begin{aligned}
\left.\left(f^{-1}(\phi)\right)^{+}\left(x^{-1}\right)\right) & =\phi^{+}\left(f\left(x^{-1}\right)\right) \\
& =\phi^{+}(f(x)) \text { as } \phi \text { is a bipolar M fuzzy group } \\
& =\left(f^{-1}(\phi)\right)^{+}(x) .
\end{aligned}
$$

Therefore $\left(f^{-1}(\phi)\right)^{+}\left(x^{-1}\right)=\left(f^{-1}(\phi)\right)^{+}(x)$

$$
\begin{aligned}
& \Leftrightarrow\left(g^{-1}(\psi)\right)^{+}\left(x^{-1}\right)=\psi^{+}\left(g\left(x^{-1}\right)\right) \\
& =\psi^{+}\left(g(x)^{-1}\right) \text { as } \mathrm{g} \text { is an M homomorphism } \\
& =\psi^{+}(g(x)) \text { as } \psi \text { is a bipolar anti M fuzzy group } \\
& =\left(g^{-1}(\psi)\right)^{+}(x) .
\end{aligned}
$$

Hence $\left(f^{-1}(\phi)\right)^{+}\left(x^{-1}\right)=\left(f^{-1}(\phi)\right)^{+}(x) \Leftrightarrow\left(g^{-1}(\psi)\right)^{+}\left(x^{-1}\right)=\left(g^{-1}(\psi)\right)^{+}(x)$.

iv) $\left.\left(f^{-1}(\phi)\right)^{-}\left(x^{-1}\right)\right)=\phi^{-}\left(f\left(x^{-1}\right)\right)$

$$
\begin{aligned}
& =\phi^{-}(f(x)) \text { as } \phi \text { is a bipolar M fuzzy group } \\
& =\left(f^{-1}(\phi)\right)^{-}(x) .
\end{aligned}
$$




$$
\begin{aligned}
& \text { Therefore }\left(f^{-1}(\phi)\right)^{-}\left(x^{-1}\right)=\left(f^{-1}(\phi)\right)^{-}(x) \\
& \qquad \begin{aligned}
\Leftrightarrow\left(g^{-1}(\psi)\right)^{-}\left(x^{-1}\right) & =\psi^{-}\left(g\left(x^{-1}\right)\right) \\
& =\psi^{-}\left(g(x)^{-1}\right) \text { as } \mathrm{g} \text { is an M homomorphism } \\
& =\psi^{-}(g(x)) \text { as } \psi \text { is a bipolar anti M fuzzy group } \\
& =\left(g^{-1}(\psi)\right)^{-}(x) .
\end{aligned}
\end{aligned}
$$

Hence $\left(f^{-1}(\phi)\right)^{-}\left(x^{-1}\right)=\left(f^{-1}(\phi)\right)^{-}(x) \Leftrightarrow\left(g^{-1}(\psi)\right)^{-}\left(x^{-1}\right)=\left(g^{-1}(\psi)\right)^{-}(x)$.

v) $\left(f^{-1}(\phi)\right)^{+}(m x)=\phi^{+}(f(m x))$

$$
\begin{aligned}
& \geq \phi^{+}(f(x)) \text { as } \phi \text { is bipolar Mfuzzy group } \\
& =\left(f^{-1}(\phi)\right)^{+}(x) .
\end{aligned}
$$

$$
\begin{aligned}
& \text { Therefore }\left(f^{-1}(\phi)\right)^{+}(m x) \geq\left(f^{-1}(\phi)\right)^{+}(x) \\
& \Leftrightarrow\left(g^{-1}(\psi)\right)^{+}(m x)=\psi^{+}(g(m x)) \\
& =\psi^{+}(m g(x)) \text { as } \mathrm{g} \text { is an } \mathrm{M} \text { homomorphism } \\
& \leq \psi^{+}(g(x)) \text { as } \psi \text { is a bipolar anti Mfuzzy group } \\
& =\left(g^{-1}(\psi)\right)^{+}(x) \text {. }
\end{aligned}
$$

Hence $\left(f^{-1}(\phi)\right)^{+}(m x) \geq\left(f^{-1}(\phi)\right)^{+}(x) \Leftrightarrow\left(g^{-1}(\psi)\right)^{+}(m x) \leq\left(g^{-1}(\psi)\right)^{+}(x)$.

vi) $\left(f^{-1}(\phi)\right)^{-}(m x)=\phi^{-}(f(m x))$

$$
\begin{aligned}
& \leq \phi^{-}(f(x)) \text { as } \phi \text { is bipolar M fuzzy group } \\
& =\left(f^{-1}(\phi)\right)^{-}(x) .
\end{aligned}
$$

Therefore $\left(f^{-1}(\phi)\right)^{-}(m x) \leq\left(f^{-1}(\phi)\right)^{-}(x) \Leftrightarrow\left(g^{-1}(\psi)\right)^{-}(m x)$

$$
\begin{aligned}
& =\psi^{-}(g(m x)) \\
& =\psi^{-}(m g(x)) \text { as } \mathrm{g} \text { is an M homomorphism } \\
& \geq \psi^{-}(g(x)) \text { as } \psi \text { is a bipolar anti M fuzzy group } \\
& =\left(g^{-1}(\psi)\right)^{-}(x) .
\end{aligned}
$$

Hence $\left(f^{-1}(\phi)\right)^{-}(m x) \leq\left(f^{-1}(\phi)\right)^{+}(x) \Leftrightarrow\left(g^{-1}(\psi)\right)^{-}(m x) \geq\left(g^{-1}(\psi)\right)^{-}(x)$.

Hence $f^{-1}(\phi)=\mu$ is a bipolar intuitionistic $\mathrm{M}$ fuzzy group of $G_{1}$ and $g^{-1}(\psi)=v$ is a bipolar intuitionistic anti M fuzzy group of $G_{1}$.

Theorem.3.7 Let $\mathrm{f}$ and $\mathrm{g}$ be an intuitionistic $\mathrm{M}$ anti homomorphism from an $\mathrm{M}$ fuzzy group of $G_{1}$ onto an $\mathrm{M}$ fuzzy group of $G_{2}$. If $\mu=\left(\mu^{+}, \mu^{-}\right)$is a bipolar intuitionistic M fuzzy group of $G_{1}$ then $f(\mu)$ the image of $\mu$ under $\mathrm{f}$ is a bipolar intuitionistic $\mathrm{M}$ fuzzy group of $G_{2}$ if and only if 
$v=\left(v^{+}, v^{-}\right)$is a bipolar intuitionistic anti M fuzzy group of $G_{1}$ then $g(v)$ the image of $v$ under $\mathrm{g}$ is a bipolar intuitionistic anti M fuzzy group of $G_{2}$.

Proof Let $f: G_{1} \rightarrow G_{2}$ and $g: G_{1} \rightarrow G_{2}$ be an intuitionistic $\mathrm{M}$ anti homomorphism and let $\mu=\left(\mu^{+}, \mu^{-}\right)$and $v=\left(v^{+}, v^{-}\right)$is a bipolar intuitionistic $\mathrm{M}$ fuzzy group and bipolar intuitionistic anti M fuzzy group of $G_{1} \cdot \mu^{+}: G_{1} \rightarrow[0,1] \& u^{-} G_{1} \rightarrow-1,0$ d $d v^{+} \quad G \rightarrow[0,1] \& G \div-$ I are mappings, to prove a bipolar intuitionistic fuzzy subset $f(\mu)=\left(f(\mu)^{+}, f(\mu)^{-}\right)$and $g(v)=\left(g(v)^{+}, g(v)^{-}\right)$on $G_{2}$ is a bipolar intuitionistic M fuzzy group and bipolar intuitionistic anti M fuzzy group.

Let $u, v \in G_{2}$ since $\mathrm{f}$ is an intuitionistic $\mathrm{M}$ anti homomorphism so there exist $x, y \in G_{1}$ such that $\mathrm{f}(\mathrm{x})=\mathrm{u}$ and $\mathrm{f}(\mathrm{y})=\mathrm{v}$, it follows that $x y \in f^{-1}(u v)$ that $\mathrm{g}$ is intuitionistic $\mathrm{M}$ anti homomorphism so there exist $x, y \in G_{1}$ such that $\mathrm{g}(\mathrm{x})=\mathrm{u}, \mathrm{g}(\mathrm{y})=\mathrm{v}$ which implies $x y \in g^{-1}(u v)$

i) Let $f(\mu)^{+}(u v) \geq \max \left\{\mu^{+}(x y): x \in f^{-1}(u), y \in f^{-1}(v)\right\}$

$$
\begin{aligned}
& \geq \max \left\{\min \left\{\mu^{+}(x), \mu^{+}(y)\right\}: x \in f^{-1}(u), y \in f^{-1}(v)\right\} \\
& =\min \left\{f(\mu)^{+}(u), f(\mu)^{+}(v)\right\} .
\end{aligned}
$$

Therefore $f(\mu)^{+}(u v) \geq \min \left\{f(\mu)^{+}(u), f(\mu)^{+}(v)\right\}$

$$
\begin{aligned}
& \Leftrightarrow\left(1-g(v)^{+}\right)(u v) \geq \min \left\{\left(1-g(v)^{+}\right)(u),\left(1-g(v)^{+}\right)(v)\right\} \\
& \Leftrightarrow g(v)^{+}(u v) \leq 1-\min \left\{\left(1-g(v)^{+}\right)(u),\left(1-g(v)^{+}\right)(v)\right\} \\
& \Leftrightarrow g(v)^{+}(u v) \leq \max \left\{g(v)^{+}(u), g(v)^{+}(v)\right\} .
\end{aligned}
$$

Hence $\left(f(\mu)^{+}\right)(u v) \geq \min \left\{f(\mu)^{+}(u), f(\mu)^{+}(v)\right\}$

$$
\Leftrightarrow g(v)^{+}(u v) \leq \max \left\{g(v)^{+}(u), g(v)^{+}(v)\right\} .
$$

ii) Let $f(\mu)^{-}(u v) \leq \max \left\{\mu^{-}(x y): x \in f^{-1}(u), y \in f^{-1}(v)\right\}$

$$
\begin{aligned}
& \leq \max \left\{\max \left\{\mu^{-}(x), \mu^{-}(y)\right\}: x \in f^{-1}(u), y \in f^{-1}(v)\right\} \\
& =\max \left\{f(\mu)^{-}(u), f(\mu)^{-}(v)\right\} .
\end{aligned}
$$

Therefore $f(\mu)^{-}(u v) \leq \max \left\{f(\mu)^{-}(u), f(\mu)^{-}(v)\right\}$

$$
\begin{aligned}
& \Leftrightarrow\left(-1-g(v)^{-}\right)(u v) \leq \max \left\{\left(-1-g(v)^{-}\right)(u),\left(-1-g(v)^{-}\right)(v)\right\} \\
& \Leftrightarrow g(v)^{-}(u v) \geq-1-\max \left\{\left(-1-g(v)^{-}\right)(u),\left(-1-g(v)^{-}\right)(v)\right\} \\
& \Leftrightarrow g(v)^{-}(u v) \geq \min \left\{g(v)^{-}(u), g(v)^{-}(v)\right\} .
\end{aligned}
$$

Hence $f(\mu)^{-}(u v) \leq \max \left\{f(\mu)^{-}(u), f(\mu)^{-}(v)\right\}$

$$
\Leftrightarrow g(v)^{-}(u v) \geq \min \left\{g(v)^{-}(u), g(v)^{-}(v)\right\} .
$$


iii) Consider $f(\mu)^{+}\left(u^{-1}\right)=\max \left\{\mu^{+}(x) ; x \in f^{-1}\left(u^{-1}\right)\right\}$

$$
\begin{aligned}
& =\max \left\{\mu^{+}\left(x^{-1}\right) ; x^{-1} \in f^{-1}(u)\right\} \\
& =f(\mu)^{+}(u) .
\end{aligned}
$$

Therefore $f(\mu)^{+}\left(u^{-1}\right)=f(\mu)^{+}(u) \Leftrightarrow\left(1-g(v)^{+}\right)\left(u^{-1}\right)=\left(1-g(v)^{+}\right)(u)$

$$
\Leftrightarrow g(v)^{+}\left(u^{-1}\right)=g(v)^{+}(u) .
$$

Hence $f(\mu)^{+}\left(u^{-1}\right)=f(\mu)^{+}(u) \Leftrightarrow g(v)^{+}\left(u^{-1}\right)=g(v)^{+}(u)$.

iv) Consider $f(\mu)^{-}\left(u^{-1}\right)=\min \left\{\mu^{-}(x) ; x \in f^{-1}\left(u^{-1}\right)\right\}=\min \left\{\mu^{-}\left(x^{-1}\right) ; x^{-1} \in f^{-1}(u)\right\}$

$$
=\left(f(\mu)^{-}\right)(u) \text {. }
$$

Therefore $f(\mu)^{-}\left(u^{-1}\right)=f(\mu)^{-}(u) \Leftrightarrow\left(-1-g(v)^{-}\right)\left(u^{-1}\right)=\left(-1-g(v)^{-}\right)(u)$

$$
\Leftrightarrow g(v)^{-}\left(u^{-1}\right)=g(v)^{-}(u) \text {. }
$$

Hence $f(\mu)^{-}\left(u^{-1}\right)=f(\mu)^{-}(u) \Leftrightarrow g(v)^{-}\left(u^{-1}\right)=g(v)^{-}(u)$.

Therefore $f(\mu)$ and $g(v)$ is a bipolar fuzzy subgroup of $G_{2}$.

v) Consider $m \in M$ and $u \in G_{2}$

$$
\begin{aligned}
f(\mu)^{+}(m u) & =\max \left\{\mu^{+}(m u) ; x \in f^{-1}(u)\right\} \geq \max \left\{\mu^{+}(x) ; x \in f^{-1}(u)\right\} \\
& =f(\mu)^{+}(u) .
\end{aligned}
$$

Therefore $f(\mu)^{+}(m u) \geq f(\mu)^{+}(u) \Leftrightarrow\left(1-g(v)^{+}\right)(m u) \geq\left(1-g(v)^{+}\right)(u)$

$$
\Leftrightarrow g(v)^{+}(m u) \leq g(v)^{+}(u) \text {. }
$$

Hence $f(\mu)^{+}(m u) \geq f(\mu)^{+}(u) \Leftrightarrow g(v)^{+}(m u) \leq g(v)^{+}(u)$.

vi ) Consider $m \in M$ and $u \in G_{2}$

$$
\begin{aligned}
f(\mu)^{-}(m u) & =\min \left\{\mu^{-}(m u) ; x \in f^{-1}(u)\right\} \leq \min \left\{\mu^{-}(x) ; x \in f^{-1}(u)\right\} \\
& =f(\mu)^{-}(u) .
\end{aligned}
$$

Therefore $f(\mu)^{-}(m u) \leq f(\mu)^{-}(u) \Leftrightarrow\left(-1-g(v)^{-}\right)(m u) \leq\left(-1-g(v)^{-}\right)(u)$

$$
\Leftrightarrow g(v)^{-}(m u) \geq g(v)^{-}(u) \text {. }
$$

Hence $f(\mu)^{-}(m u) \leq f(\mu)^{-}(u) \Leftrightarrow g(v)^{-}(m u) \geq g(v)^{-}(u)$.

Hence if $\mu$ be a bipolar intuitionistic M fuzzy group of $G_{1}$ then $f(\mu)$ is a bipolar M fuzzy group of $G_{2}$ if and only if $v$ be a bipolar anti M fuzzy group of $G_{1}$ then $g(v)$ be a bipolar intuitionistic anti M fuzzy group of $G_{2}$. 


\section{Conclusion}

The concept of a bipolar intuitionistic M fuzzy group is a new algebraic structure of a bipolar intuitionistic $M$ fuzzy subgroup of a $M$ fuzzy group and anti $M$ fuzzy group are defined and some related properties are investigated. The purpose of the study is to implement the fuzzy set theory and group theory of bipolar intuitionistic $M$ fuzzy subgroup of a $M$ fuzzy group and anti $M$ fuzzy group. The relation between of a bipolar intuitionistic $M$ fuzzy group and bipolar intuitionistic anti $M$ fuzzy group are established. We hope that our results can also be extended to other algebraic system.

\section{References}

[1]. K. Chakrabarthy, Biswas R. Nanda, A note on union and intersection of intuitionistic fuzzy sets, Notes on intuitionistic fuzzy sets, 3(4), 1997.

[2]. P.S. Das, Fuzzy groups and level subgroups, Journal of Mathematical Analysis and its Application, 84, 1981, $264-269$.

[3]. V.N. Dixit., A. Rajeshkumar, Naseem Ajmal, Level subgroups and union of fuzzy subgroups, Fuzzy sets and systems, 37, 1990, 359-371.

[4]. Krassimir T. Atanassov, Intuitionistic fuzzy sets :Theory and Application (Physica-verlag, A springer - verlag company)

[5]. K.. M. Lee, Bipolar valued fuzzy sets and their operations, proceeding of International conference on Intelligent Technologies, Bangkok, Thailand, 2000, 307-312.

[6]. Mourad Oqla MASSA'DEH, Structure properties of an intuitionistic anti fuzzy M subgroups, mathematics section.

[7]. R. Muthura, M. Sridharan, Bipolar anti fuzzy HX groups and its lower level sub HX groups, Journal of physical sciences, 16, 2012, 157-169.

[8]. R. Muthuraj, M. Rajinikannan, M. S. Muthuraman, The M- homomorphism and M-anti homomorphism of an M fuzzy subgroups and its lower level subgroups, International journal of computer applications 2(1), 2010, 66-70.

[9]. R. Muthuraj, M. Sridharan, Homomorphism and anti homomorphism of bipolar fuzzy sub HX groups, General mathematical notes, 17( 2), 2013, 53-65.

[10]. N. Palaniappan, R. Muthuraj, Anti fuzzy group and lower level subgroups, Antarctica Journal of Mathematics, 1(1), 2004, 71-76.

[11]. A. Rajeshkumar, Fuzzy Algebra: volume I (Publication division, university of Delhi)

[12]. A. Rosenfeld, Fuzzy Groups, Journal of Mathematical Analysis and its Application (35), 1971, $512-517$

[13]. L.A. Zadeh, Fuzzy sets, information and control, 8, 1965, 338-353.

[14]. H.J. Zimmermann, Fuzzy set theory and its Applications (Kluwer - Nijhoff publishing co., 1985).

[15]. W.R. Zhang, Bipolar fuzzy sets, Proceeding of FUZZ - IEEE, 1998, 835-840. 